\title{
Post-operative neck ultrasound and risk stratification in differentiated thyroid cancer patients with initial lymph node involvement
}

\author{
Charlotte Lepoutre-Lussey ${ }^{1, *}$, Dina Maddah ${ }^{1, *}$, Jean-Louis Golmard ${ }^{2}$, Gilles Russ ${ }^{1}$, \\ Frédérique Tissier ${ }^{3}$, Christophe Trésallet ${ }^{4}$, Fabrice Menegaux ${ }^{4}$, André Aurengo ${ }^{1}$ and \\ Laurence Leenhardt ${ }^{1}$ \\ Departments of ${ }^{1}$ Nuclear Medicine, ${ }^{2}$ Biostatistics, ${ }^{3}$ Pathology and ${ }^{4}$ Endocrine Surgery, Institute of Cancer, \\ Pitie-Salpetriere Hospital, Pierre et Marie Curie University, 47-83 Bd de l'hôpital, 75013 Paris, France \\ *(C Lepoutre-Lussey and D Maddah contributed equally to this work)
}

\author{
Correspondence \\ should be addressed \\ to $L$ Leenhardt \\ Email \\ laurence.leenhardt@ \\ psl.aphp.fr
}

\begin{abstract}
Objective: Cervical ultrasound (US) scan is a key tool for detecting metastatic lymph nodes (N1) in patients with papillary thyroid cancer (PTC). N1-PTC patients are stratified as intermediate-risk and high-risk (HR) patients, according to the American Thyroid Association (ATA) and European Thyroid Association (ETA) respectively. The aim of this study was to assess the value of post-operative cervical US (POCUS) in local persistent disease (PD) diagnosis and in the reassessment of risk stratification in N1-PTC patients.

Design: Retrospective cohort study.

Methods: Between 1997 and 2010, 638 N1-PTC consecutive patients underwent a systematic POCUS. Sensitivity, specificity, negative predictive value (NPV), and positive predictive value (PPV) of POCUS for the detection of PD were evaluated and a risk reassessment using cumulative incidence functions was carried out.

Results: After a median follow-up of 41.6 months, local recurrence occurred in 138 patients (21.6\%), of which 121 were considered to have PD. Sensitivity, specificity, NPV, and PPV of POCUS for the detection of the 121 PD were 82.6, 87.4 95.6, and $60.6 \%$ respectively. Cumulative incidence of recurrence at 5 years was estimated at $26 \%$ in ETA HR patients, $17 \%$ in ATA intermediate-risk patients, and $35 \%$ in ATA HR patients respectively. This risk fell to 9,8 , and $11 \%$ in the above three groups when the POCUS result was normal and to $<6 \%$ when it was combined with thyroglobulin results at ablation.

Conclusion: POCUS is useful for detecting PD in N1-PTC patients and for stratifying individual recurrence risk. Its high NPV could allow clinicians to tailor follow-up recommendations to individual needs.
\end{abstract}

\section{Introduction}

In patients with papillary thyroid cancer (PTC), initial lymph node (LN) involvement (N1) is frequent (50-70\% of cases) (1). Although the disease-specific survival rate is good, exceeding $90 \%$ at 10 years $(1,2)$, the outcome for N1-PTC patients is variable, with a risk of locoregional recurrence and persistent disease (PD) varying from 2 to $40 \%(3,4,5)$. The risk of recurrence of N1-PTC is classified to be intermediate in the American Thyroid Association
(ATA) guidelines (6) and to be high by the European Thyroid Association (ETA) (7) and French (SFE) societies (8). This risk stratification is based on the pathological tumor node metastasis (pTNM) classification. Recent reports have proposed individual risk stratification based on data obtained 8-12 months after RAI $(5,9)$, while others outline the utility of a post-operative staging using stimulated thyroglobulin (s-Tg) tests to rule out PD after (c) 2014 European Society of Endocrinology Printed in Great Britain
Published by Bioscientifica Ltd. 
surgery $(10,11,12)$. Neck ultrasonography (US) is the most sensitive method for detecting cervical LN metastasis $(1,13)$ and its usefulness just before surgery and 6-12 months after radioiodine ablation (RAI) is well established (6). This paper presents further evidence regarding the interest of post-operative cervical US (POCUS) in the management of these patients as recently highlighted (14). The objective of this retrospective study was to assess the value of POCUS in the detection of PD in N1-PTC patients, and to compare its value with postoperative s- $\mathrm{Tg}$ (RAI-Tg) and post-therapeutic whole-body scans (tWBS). Our hypothesis is that POCUS could be useful in redefining the initial recurrence risk based on the initial pTNM staging and thus enable clinicians to tailor RAI and follow-up recommendations to individual needs.

\section{Subjects and methods}

\section{Patients}

Between 1997 and 2010, 731 N1-PTC patients were consecutively referred to our center for RAI after thyroidectomy, and systematically underwent a POCUS examination. Their medical records were retrospectively reviewed.

Ninety-three patients were excluded: 75 without follow-up in our institution, eight without examination during the first 6 months after radioiodine therapy, four with no primary tumor discovered (Tx), and six with unavailable data. Adequate clinico-pathological and follow-up records of the remaining 638 patients were analyzed. The clinical characteristics of the patients are given in Table 1.

\section{Initial surgery, pTNM staging, and risk stratification}

Among our patients, 324 (50.6\%) had undergone initial thyroidectomy at our institution (C Trésallet and F Menegaux) and 314 at other centers, and were then referred to our department. All tumors were classified using the 2010 pTNM scoring system (Table 1). The surgical procedure was guided by clinical and imaging findings, including at least a systematic preoperative cervical ultrasound. All patients had total thyroidectomy and $97 \%$ of them had LN dissection (LND). The extent of the LND took into account the clinical and preoperative ultrasound (US) data and remained at the discretion of the attending surgeon.

All patients had LN involvement, discovered either at systematic LND or fortuitously at histological
Table 1 Characteristics of patients $(n=638)$.

\begin{tabular}{l} 
Age (median (range)) \\
Sex (females/males (\%)) \\
Thyroidectomy \\
Total \\
Subtotal \\
LN excision (\%) \\
Central (C) \\
Lateral (L) \\
C+L \\
No LN dissection \\
pTNM stage (\%) \\
pT1 \\
pT2 \\
pT3 \\
pT4 \\
Multifocality (\%) \\
Vascular invasion (\%) \\
Primary tumor extrathyroidal \\
extension (ETE) (\%) \\
LN involvement (\%) \\
N1a \\
N1b \\
Unspecified \\
Extra-capsular LN involvement (LN-ECI) \\
Yes \\
No \\
Unspecified \\
LN score \\
High-risk N1 (HR-N1) \\
Low-risk N1 (LR-N1) \\
Radioiodine ablation (RAI) (\%) \\
Median follow-up (months) (range) \\
\hline
\end{tabular}

$42.2(11.3-82.2)$

$482 / 156(75.5 / 24.4)$

633 (99.2)

$5(0.8)$

106 (16.6)

75 (11.8)

438 (68.6)

19 (3)

247 (38.71)

81 (12.70)

$292(45.77)$

18 (2.82)

$320(50.16)$

$70(10.97)$

275 (43.17)

289 (45.3)

345 (54.1)

$4(0.6)$

208 (32.6)

361 (56.6)

$69(10.8)$

408 (64)

230 (36)

$638(100)$

$41.6(6-171)$

LR-N1, N1a patients without LN-ECl; HR-N1, N1b patients and N1a patients with LN-ECI.

examination in 97 and $3 \%$ of cases respectively (Table 1), and were thus considered to be at a high risk (HR) of recurrence as stated by the ETA consensus (7). According to the ATA guidelines, patients were considered to be at a HR of recurrence (macroscopic tumor invasion and/or incomplete tumor resection and/or distant metastases) in $49.4 \%$ of the cases $(315 / 638$ patients) or an intermediate risk ( $\mathrm{N} 1$ without the above-mentioned criteria) in 50.6\% of the cases (323/638 patients). Because of the retrospective nature of the study and the many changes in pTNM classification, we could not isolate the subgroup of microscopic invasion of tumor into the perithyroidal soft tissues at initial surgery. Consequently, we decided by default to classify all pT3 patients as ATA HR.

The location of the metastatic LN and the presence of an extra-capsular LN involvement (N1-ECI) were recorded when available. We studied the relevance of dividing the patients into two subgroups of LN involvement (LN score): a low-risk (LR) subgroup (LR-N1) including N1a patients without N1-ECI vs a HR subgroup (HR-N1) represented by 
all others (i.e. N1b patients and N1a patients with N1-ECI). Patients with unspecified location and/or unspecified N1-ECI were classified by default in the LR-N1 group.

\section{Post-operative cervical ultrasonography}

POCUS was systematically and prospectively performed for all patients after surgical treatment and just before RAI. It was performed with a real-time US scanner (Toshiba (Tokyo, Japan) or Siemens (Munich, Germany)) with a 7.5-13 MHz bandwidth linear transducer and by experienced sonographers trained in neck US. The examination included both the central and lateral neck compartments, studied with gray-scale and color Doppler. Abnormal LNs were systematically drawn onto a diagram of the neck compartments as recommended by the European guidelines (14). All US scan images were reviewed by a single experienced sonographer who was unaware of the final outcome (L Leenhardt).

According to the recent US European guidelines (14), the US result was considered suspicious when at least one of the following features was detected:

i) a vascular hypoechogenic mass in the thyroid bed, with or without microcalcifications or cystic areas,

ii) a LN with at least one of the following signs: microcalcifications, cystic areas, peripheral or anarchic vascularization, echogenicity similar to thyroid tissue (hyperechoic area).

The US examination was considered normal in the absence of these signs (14). The location (central or lateral neck) and the size (small (S) and long axis (L)) of the LN were recorded. Criteria of intermediate significance such as L:S ratio $<2$ and absence of hilum were not considered suspicious in the absence of the four major echo-Doppler signs aforementioned. In cases of multiple suspicious LN, the largest was analyzed.

\section{RAl treatment and follow-up}

All patients received an ablative ${ }^{131} \mathrm{I}$ dose (RAI) of $3.7 \mathrm{GBq}$ within 12 months of surgery, on average, 90 days after surgery ( \pm 48 days).

Tg levels were measured using the immunoradiometric technique, BRAHMS, coupled with the systematic detection of anti-Tg antibodies. s-Tg levels were assessed at the time of radioiodine ablation (RAI-Tg) after 4 weeks of thyroid hormone withdrawal or after stimulation by rTSH (Thyrogen) during the follow-up. An RAI-Tg value $>10 \mu \mathrm{g} / \mathrm{l}$ was considered suspicious for persistent or recurrent disease (12). Patients with interfering anti-Tg antibodies were not excluded, as our aim was to assess the POCUS value in all N1-PTC patients.

A WBS was performed 5 days after administration of the ablative ${ }^{131}$ I dose of $3.7 \mathrm{GBq}$ (tWBS). Images were obtained using an APEX HELIX SPX camera (Elscint, Nicosia, Cyprus) until 2007 and a TDM INFINIA camera (GE Medical System) afterwards. Radioiodine uptake outside the thyroid bed was considered suspicious for metastatic LN persistence or early recurrence.

Median follow-up was 41.6 months (range 6-171 months). Follow-up protocol consisted of a systematic first check-up 6 months after radioiodine ablation, including clinical examination, neck ultrasonography, and s-Tg (rTSH $\mathrm{Tg}$ or Tg level after levothyroxine (L-T ${ }_{4}$ ) withdrawal). A WBS after a diagnostic ${ }^{131} \mathrm{I}$ dose (dWBS) under thyroidstimulating hormone (TSH) stimulation was also performed for 378 patients at 6 months (58\%). For 59 patients (9\%), it was the only imaging examination performed at 6 months (without cervical ultrasonography). Subsequent check-ups were systematically performed under TSH stimulation 12 months later and every 3 years for 7 years and subsequently, under suppressive treatment, every 3 years (on site or via periodic correspondence with patients or their referring physicians). Other imaging examinations (cervical computed tomography, ${ }^{18}$ FDG positron emission tomography) were prescribed at the discretion of the attending physician. All patients were receiving TSH suppressive therapy. Follow-up information and date of death were obtained either from the medical records or from town council registers.

\section{Clinical endpoints}

The diagnosis of recurrence was assessed by histological analysis after surgery, by fine-needle aspiration biopsy (FNAB; malignant cytological result and/or Tg washout measurement $>10 \mu \mathrm{g} / \mathrm{l}(15))$, or positive ${ }^{131}$ I cervical uptake after a ${ }^{131}$ I diagnostic (11.1 MBq) or therapeutic (3.7 GBq) dose. WBS was considered abnormal, suspicious for recurrence, when uptake was seen outside the thyroid bed at ablation or if cervical uptake was persistent after the first radioiodine treatment (dWBS and/or tWBS at second treatment).

Recurrence was divided into two groups depending on the date of occurrence (Fig. 1):

i) Persistence (PD) if disease was detected at ablation and/or at 6 months of follow-up without documented disease-free period.

ii) Late recurrence (1-RD) if no PD was detected and when recurrence was detected after a normal check-up at 6 months (negative cervical US, s-Tg $\leq 1 \mu \mathrm{g} / \mathrm{l}$ (and/or 


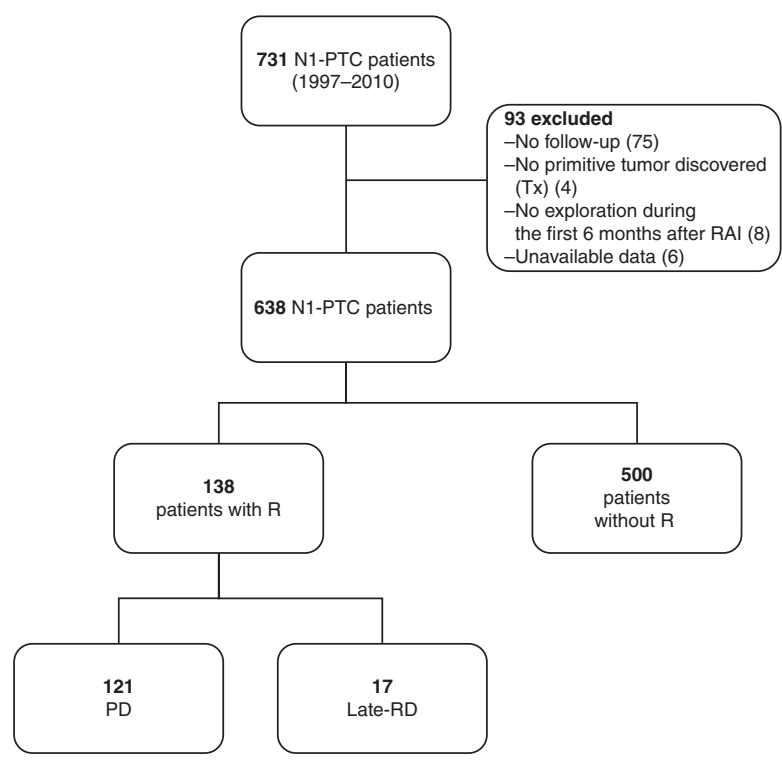

\section{Figure 1}

Study population. N1-PTC, papillary thyroid cancer with initial lymph node involvement; $R$, locoregional recurrence;

PD, locoregional persistent disease.

in case of detectable anti-Tg antibody, if the d-WBS was normal)).

The POCUS was performed, on average, 90 days $( \pm 48$ days) after surgery. Its diagnostic value was calculated taking only PD into account and regarding the entire follow-up of the patient. In this analysis, POCUS results were considered:

i) True positive (TP) if POCUS and other cervical US performed during the follow-up were suspect for the same structure until the PD or l-RD evidence.

ii) True negative if POCUS and all other cervical US performed during the follow-up were normal without recurrence and/or when the l-RD occurred tardily after more than 1-year follow-up with both POCUS and 6-month cervical US (US-6) negative.

iii) False positive (FP) if POCUS was suspect without recurrence during the entire follow-up of the patient.

iv) False negative if POCUS was normal with PD or l-RD occurrence detected as a result of other cervical US and/or dWBS and/or tWBS after a second RAI treatment during the first year of follow-up.

\section{Statistical analysis}

Descriptive statistics used numbers and percentages for qualitative variables; mean and s.D.s or medians and interquartile intervals for quantitative ones. The diagnostic value of POCUS, RAI Tg, and tWBS was evaluated retrospectively according to PD occurrence.

The interest of POCUS for PD was first evaluated using the usual parameters: sensitivity, specificity, negative predictive value (NPV), and positive predictive value (PPV). Then, a complete analysis of the diagnostic factors of recurrence was carried out, with $\chi^{2}$-tests or Fisher's exact tests for qualitative variables and Student's $t$-tests for quantitative ones, followed by a stepwise logistic regression. Variables were entered into the stepwise regression when their $P$ value was $<0.10$ in the univariate analysis, and they were retained in the final model when the $P$ value of the Wald test was $<0.05$. The relationships between sensitivity or specificity and the timing of POCUS and location of abnormal findings were also assessed using univariate and bivariate logistic regressions. Cumulative incidence of recurrence functions was estimated by one-minus the Kaplan-Meier survival function estimate. For late recurrence and overall survival, two survival analyses were carried out with the same method: first, a univariate analysis of each potential prognostic factor, involving log-rank tests for qualitative variables and univariate Cox models for quantitative ones. In a second step, variables with a $P$ value lower than 0.10 in the univariate step were entered in a stepwise Cox model regression. Variables significant with a $P$ value lower than 0.05 with the Wald tests were retained in the final models. All the tests were two sided. Computations were carried out using the SAS V9 statistical package (SAS, Cary, NC, USA).

\section{Results}

Recurrence occurred in 138 patients (21.6\%), who were divided into two groups: 121 PD (88\%) and 17 l-RD (12\%). Recurrence was diagnosed by histology for 116 patients (86\%), positive ${ }^{131}$ I cervical uptake for 13 patients $(9.6 \%)$, and FNAB for nine patients (5\%). The average time period between the primary tumor diagnosis and the recurrence onset was 18 months (range 3-144 months; s.D. 17.7). Among the $121 \mathrm{PD}, 26$ were located in the central neck compartment only, 63 in the lateral neck, and 32 concerned both the central and lateral compartments.

\section{Diagnostic value of POCUS and comparison with tWBS and RAI-Tg}

For the detection of PD, sensitivity, specificity, NPV, and PPV of POCUS were 82.6, 87.4, 95.6, and $60.6 \%$ respectively. Data are detailed in Table 2. 
Table 2 Risk factor for locoregional persistent disease (PD).

\begin{tabular}{|c|c|c|c|c|c|}
\hline \multirow[b]{2}{*}{ Characteristics } & \multicolumn{2}{|c|}{ PD } & \multirow{2}{*}{$\frac{\text { Univariate analysis }}{P \text { value }}$} & \multicolumn{2}{|c|}{ Multivariate analysis } \\
\hline & Yes $(n=121)$ & No $(n=517)$ & & OR $(95 \% \mathrm{Cl})$ & $P$ value \\
\hline Age $(n(\%))$ & & & 0.91 & - & - \\
\hline$\geq 45$ years & $54(44.6)$ & $228(44.1)$ & & & \\
\hline$<45$ years & $67(55.4)$ & $289(55.9)$ & & & \\
\hline $\operatorname{Sex}(n(\%))$ & & & 0.0073 & NS & NS \\
\hline $\mathrm{M}$ & 41 (33.9) & 115 (22.2) & & & \\
\hline $\mathrm{F}$ & $80(66.1)$ & $402(77.8)$ & & & \\
\hline LN dissection $(n(\%))$ & & & 0.27 & - & - \\
\hline Central (C) & 15 (12.4) & 91 (18.3) & & & \\
\hline Lateral (L) & $14(11.6)$ & $61(12.2)$ & & & \\
\hline$C+L$ & $92(76)$ & 346 (69.5) & & & \\
\hline Multifocal involvement $(n(\%))$ & & & 0.002 & NS & NS \\
\hline Yes & $76(62.8)$ & $244(47.2)$ & & & \\
\hline No & $45(37.2)$ & $273(52.8)$ & & & \\
\hline Vascular invasion (n (\%)) & & & 0.06 & NS & NS \\
\hline Yes & $19(15.7)$ & $51(9.9)$ & & & \\
\hline No & $102(84.3)$ & $466(90.1)$ & & & \\
\hline ETE primary tumor $(n(\%))$ & & & $<0.0001$ & NS & NS \\
\hline Yes & $75(62)$ & $200(38.8)$ & & & \\
\hline No & $46(38)$ & $316(61.2)$ & & & \\
\hline $\begin{array}{l}\text { Tumor size }(\mathrm{mm}), \text { mean } \pm \text { s.D. } \\
\text { pTx classification }(n(\%))\end{array}$ & $23.6 \pm 17.8$ & $18.1 \pm 12.1$ & $\begin{array}{l}<0.0001 \\
<0.0001\end{array}$ & NS & NS \\
\hline pT1 & $28(23.1)$ & $219(42.4)$ & & NS & NS \\
\hline pT2 & $13(10.7)$ & $68(13.2)$ & & NS & NS \\
\hline pT3 & $70(57.9)$ & $222(42.9)$ & & NS & NS \\
\hline pT4 & $10(8.3)$ & $8(1.5)$ & & NS & NS \\
\hline LN involvement $(n(\%))$ & & & $<0.0001$ & NS & NS \\
\hline N1a & $34(28.1)$ & $255(49.7)$ & & & \\
\hline N1b & $87(71.9)$ & $258(50.3)$ & & & \\
\hline LN-ECI $(n(\%))$ & & & $<0.0001$ & NS & NS \\
\hline Yes & $65(53.7)$ & $143(27.7)$ & & & \\
\hline No & 47 (38.8) & $314(60.7)$ & & & \\
\hline Unspecified & $9(7.5)$ & $60(11.6)$ & & & \\
\hline LN score & & & $<0.0001$ & NS & NS \\
\hline High-risk N1 (HR-N1) & $103(85.1)$ & $305(59)$ & & & \\
\hline Low-risk N1 (LR-N1) & $18(14.9)$ & $212(41)$ & & & \\
\hline Initial distant metastasis $(n(\%))$ & & & 0.04 & NS & NS \\
\hline MO & $114(94.2)$ & $505(97.7)$ & & & \\
\hline M1 & $7(5.8)$ & $12(2.3)$ & & & \\
\hline POCUS $(n(\%))$ & & & $<0.0001$ & & $<0.0001$ \\
\hline Abnormal result & $100(82.6)$ & $65(12.6)$ & & $14.21(7.0-28.83)$ & \\
\hline Normal result & $21(17.4)$ & $452(87.4)$ & & 1 & \\
\hline tWBS $(n(\%))$ & & & $<0.0001$ & NS & NS \\
\hline Abnormal result & $48(39.7)$ & $101(19.5)$ & & & \\
\hline Normal result & $73(60.3)$ & $416(80.5)$ & & & \\
\hline RAI-Tg (n (\%)) & & & & & $<0.0001$ \\
\hline$>10 \mu \mathrm{g} / \mathrm{l}$ & $83(68.6)$ & $92(17.8)$ & $<0.0001$ & $5.71(3.25-10.04)$ & \\
\hline$<10 \mu \mathrm{g} / \mathrm{l}$ & $38(31.4)$ & $425(82.2)$ & & 1 & \\
\hline
\end{tabular}

C, central; L, lateral; ETE, extrathyroidal extension; LN-ECI, extra-capsular LN involvement; RAI-Tg, thyroglobulin at radioiodine ablation.

The location of abnormal findings (central or lateral neck) did not significantly influence POCUS sensitivity and specificity even when correlated with the timing of POCUS $(P=0.74)$.

The timing of POCUS alone did not significantly influence its sensitivity ( $P=0.91)$; however, the specificity increased with the time lapse between POCUS and surgery and exceeded 90\% when performed 90 days after surgery (Table 3).

POCUS revealed LN metastasis (i.e. a TP POCUS result) in 100/638 (15.7\%) patients, among which 58 were located in the central neck (exclusively or with associated lateral involvement). The mean size of suspect $\mathrm{LN}$ was 16.3 $( \pm 7.1) \mathrm{mm}$ for the largest $(\mathrm{L})$ diameter and $8.8( \pm 3.8) \mathrm{mm}$ 
Table 3 Sensitivity and specificity of POCUS according to the timing for POCUS after surgery.

\begin{tabular}{|c|c|c|c|c|c|}
\hline & \multicolumn{5}{|c|}{$\begin{array}{c}\text { Time period between surgery } \\
\text { and POCUS (days) }\end{array}$} \\
\hline & $<60$ & $60-90$ & $90-120$ & $>120$ & $P$ value \\
\hline Sensitivity (\%) & 78 & 92 & 79 & 80 & 0.91 \\
\hline Specificity (\%) & 78 & 86 & 91 & 92 & 0.0039 \\
\hline
\end{tabular}

for the smallest (S) diameter. These patients with TP POCUS were treated for their PD, on average, 15 months after the first surgery and 13 months after the POCUS.

Sixty-five patients (10\%) had a suspect POCUS without recurrence and were thus considered to have FP results. Their characteristics and outcomes are summarized in Table 4. Among these 65 FP POCUS patients, 26 had an abnormal tWS and the median RAI-Tg was $52 \mu \mathrm{g} / \mathrm{l}$ (Table 4 ).

Finally, 473 POCUS patients (71\%) were considered normal and 452 of them (95.6\%) never developed a recurrence. Among the total series, 21 POCUS results were considered false negative (FN, 3.3\%). The POCUS was normal for all 21 patients, but for 16 of them the subsequent US control performed during the first year of follow-up revealed a suspicious LN (cytologically or histologically proven) and for five of them a positive ${ }^{131} \mathrm{I}$ cervical uptake after a second radioiodine treatment was observed. Five patients among the 21 FN POCUS had an abnormal cervical tWBS uptake at ablation. For two of these cases, PD was diagnosed after a positive ${ }^{131}$ I cervical uptake and for three of them PD was proven histologically.

An uptake outside the thyroid bed was detected at tWBS in 149/638 patients. Among these, 48 patients had PD proven by histology, cytology, or persistence on tWBS after a second RAI treatment. The remaining 101 patients with abnormal tWBS were considered FP of tWBS in the absence of PD or proven recurrence during subsequent follow-up. Seventy-three patients with normal cervical tWBS uptake had at least one LN metastasis detected, thanks to POCUS. Sensitivity, specificity, NPV, and PPV of tWBS for the diagnosis of PD were 39.7, 80.5, 85, and 32\% respectively.

At ablation, RAI-Tg was undetectable in 79 patients (12.4\%), under 1 and $10 \mu \mathrm{g} / 1$ in $206(32.3 \%)$ and 463 (72.6\%) patients respectively. Median RAI-Tg was $52 \mu \mathrm{g} / 1$ (range 0.15-300) and $13 \mu \mathrm{g} / \mathrm{l}$ (range 0.15-300) in FP and FN POCUS patients respectively. One hundred and seventy-one patients (27\%) had both a RAI-Tg under $1 \mu \mathrm{g} / 1$ and a negative POCUS. Among them, tWBS was abnormal in 28 cases and a locoregional recurrence was

detected in six cases: two patients with PD and four with l-RD. A RAI-Tg higher than $10 \mu \mathrm{g} / \mathrm{l}$ was chosen as a cut-off value with a good prediction of PD $(P<0.0001)$. Sensitivity, specificity, NPV, and PPV of RAI-Tg $\geq 10 \mu \mathrm{g} / \mathrm{l}$ for prediction of PD were 68.6, 82.2, 91.8, and $47.4 \%$ respectively.

Sensitivity and specificity of POCUS were significantly higher than those of tWBS $(P<0.0001$ and $P=0.0008$ respectively) and of RAI-Tg $(P=0.001$ and $P=0.01$ respectively).

\section{Predictive factors for PD, late recurrence, and patients' outcome}

In N1 PTC patients, only a suspect POCUS result and a RAI-Tg $\geq 10 \mu \mathrm{g} / \mathrm{l}$ were significantly predictive of PD $(P<0.0001)$ at multivariate analysis (Table 2$)$. None of the other factors listed on Table 2 were significant.

Table 4 Characteristics of patients with false-positive (FP) POCUS.

Total numbers

US normalization at 6 months (US-6) among FP

Number of patients with repeated cervical US (\%)

Among FP patients

Among the entire cohort

Number of FNAB (\%)

Among FP patients

Among the entire cohort

Number of negative reoperation (\%)

Among FP patients

Among the entire cohort

Number of additional RAI (\%)

Among FP patients

Among the entire cohort

Number of patients with abnormal tWBS (\%)

For all

For patients with normal US-6

For patients with abnormal US-6

Median RAI-Tg $(\mu \mathrm{g} / \mathrm{l})$ (range)

For all

For patients with normal US-6

For patients with abnormal US-6

Suspect LN characteristic

LN with microcalcification (\%)

LN with peripheral or anarchic

vascularization (\%)

LN with cystic change (\%)

Hyperechogenic LN (\%)

Endpoint

Cured (\%)

Progressive disease (\%)

Not cured not progressive (\%)
$65 / 638(10 \%)$

$38 / 65(58 \%)$

$28 / 65(43 \%)$

$28 / 638(4.3 \%)$

$11 / 65(17)$

$11 / 638(1.7)$

$2 / 65(3)$

$2 / 638(0.3 \%)$

$8 / 65(12)$

$8 / 638(1.2)$

26/65 (40)

$7 / 65(11)$

$19 / 65$ (29)

$52(0.15-300)$

$19(0.15-84.1)$

$77(0.2-300)$

18 (28)

46 (72)

$13(20)$

18 (28)

$46 / 65(70)$

2/65 (3)

$16 / 65(26)$ 
Only three factors were predictive of late recurrence (1-RD) in the univariate analysis: RAI-Tg $\geq 10 \mu \mathrm{g} / 1$ $(P=0.0091)$, initial N1b status $(P=0.0191)$, and initial M1 status $(P=0.0131)$. Only RAI-Tg $\geq 10 \mu \mathrm{g} / \mathrm{l}$ remained predictive in the multivariate analysis (HR 3.353, 95\% CI (1.278-8.793); $P=0.0139$ ).

At the end of the study, $626(98.1 \%)$ patients were still alive and 492 patients (77.1\%) considered cured: normal neck US and a s-Tg level $\leq 1 \mu \mathrm{g} / \mathrm{l}$ (and/or a normal d-WBS in case of detectable anti-Tg antibody). Among the total series, 12 (1.9\%) patients died; seven (1.1\%) of these were from their thyroid cancer and two from another cause. The cause of death was unknown for three patients. All those patients who died from their thyroid cancer had developed a recurrence. Overall survival of the entire cohort was superior by $99 \%$ at 5 years. Factors significantly influencing survival in univariate analysis were age $\geq 45$ years $(98.7 \%$ of 5 -year survival vs $100 \% ; \quad P=0.0021)$, pT4 initial stage $(P<0.0001)$, the occurrence of recurrence (HR 5.085, 95\% CI (1.25-20.67), $P=0.018)$ even in the case of persistence (PD) (97.4 vs $100 \%, P$ 0.005). In the multivariate analysis, only age at diagnosis $\geq 45$ years (HR 8.841, 95\% CI (1.05-74.28); $P=0.048)$ and pT4 initial stage still influence the survival (HR 8.062, 95\% CI (2.03-32.02); $P=0.003)$.

\section{Risk stratification}

Among the recurrence occurred in 138 patients, cumulative incidence estimated by the Kaplan-Meier method was $11.4 \%$ (69 patients) during the first year of follow-up, $22 \%$ (119 patients) within 3 years, and 26.2\% (131 patients) within 5 years.

Taking into account the initial pTNM risk stratification, $26 \%$ of ETA HR patients, $17 \%$ of ATA intermediate risk patients, and $35 \%$ of ATA HR patients developed a recurrence at 5 years (Table 5).

When the recurrence risk was reassessed with POCUS and its result was normal, only $9 \%$ of ETA HR patients, $8 \%$ of ATA intermediate, and $11 \%$ of ATA HR patients had locoregional recurrence at 5 years.

Patients with a RAI-Tg value under $10 \mu \mathrm{g} / \mathrm{l}$ also had a lower risk of recurrence estimated at 5 years, which represented $13 \%$ for ETA HR, and 9 and $18 \%$ for ATA intermediate and HR patients respectively.

Finally, a combination of normal POCUS and RAI-Tg value under $10 \mu \mathrm{g} / \mathrm{l}$ conferred a reassessed risk of recurrence lower than $6 \%$ for the three groups (Table 5).
Table 5 Five-year cumulative incidence (\%) of recurrence according to ATA and ETA initial stratification depending on POCUS and RAI-Tg results.

\begin{tabular}{|c|c|c|c|}
\hline & \multicolumn{2}{|c|}{ ATA } & \multirow{2}{*}{$\begin{array}{c}\text { ETA } \\
\text { High } \\
(n=638)\end{array}$} \\
\hline & $\begin{array}{l}\text { Intermediate } \\
\quad(n=323)\end{array}$ & $\begin{array}{l}\text { High } \\
(n=315)\end{array}$ & \\
\hline $\begin{array}{l}\text { 5-year cumulative incidence } \\
\text { of recurrence }\end{array}$ & $17 \%$ & $35 \%$ & $26 \%$ \\
\hline \multicolumn{4}{|l|}{$\begin{array}{l}\text { Risk reassessment of } \\
\text { recurrence depending } \\
\text { on POCUS result }\end{array}$} \\
\hline Normal POCUS $(n=473)$ & $8 \%$ & $11 \%$ & $9 \%$ \\
\hline Abnormal POCUS $(n=165)$ & $56 \%$ & $77 \%$ & $70 \%$ \\
\hline \multicolumn{4}{|l|}{$\begin{array}{l}\text { Risk reassessment of } \\
\text { recurrence depending } \\
\text { on Tg IRA result }\end{array}$} \\
\hline $\operatorname{Tg}$ IRA $<10 \mu \mathrm{g} / \mathrm{l}(n=463)$ & $9 \%$ & $18 \%$ & $13 \%$ \\
\hline Tg IRA $\geq 10 \mu \mathrm{g} / \mathrm{l}(n=175)$ & $42 \%$ & $64 \%$ & $55 \%$ \\
\hline \multicolumn{4}{|l|}{$\begin{array}{l}\text { Risk reassessment of } \\
\text { recurrence depending on } \\
\text { POCUS and RAI-Tg result }\end{array}$} \\
\hline $\begin{array}{l}\text { Normal POCUS and RAI-Tg } \\
<10 \mu \mathrm{g} / \mathrm{l}(n=387)\end{array}$ & $4 \%$ & $6 \%$ & $5 \%$ \\
\hline $\begin{array}{l}\text { Abnormal POCUS or } \\
\text { RAl-Tg } \geq 10 \mu \mathrm{g} / \mathrm{l}(n=251)\end{array}$ & $42 \%$ & $63 \%$ & $54 \%$ \\
\hline
\end{tabular}

Recurrence: locoregional recurrence including persistent disease (PD) and late recurrence (I-RD).

\section{Discussion}

Over the past few years, new concepts have emerged concerning the usefulness of neck ultrasonography in the management of PTC patients. They focus on three main points:

i) Neck US as the best diagnostic tool for detecting recurrence when combined with $\mathrm{Tg}$ measurement. In particular, it gives a more accurate diagnosis than a diagnostic WBS $(16,17)$.

ii) Neck US as a two-stage dynamic risk assessment for recurrence: first at the post-operative period to tailor radioiodine ablation indications to individual needs, secondly during the follow-up (especially the first 2 years) to categorize response to therapy.

iii) The concept that N1 patients have a heterogeneous risk of recurrence and that specific characteristics of LN metastases could be used to stratify the risk of recurrence in PTC.

\section{Comparison of POCUS with RAI-Tg and tWBS}

Our results showed that POCUS accuracy is better than that achieved by tWBS. This could partly be due to the fact 
that before 2007, most patients were explored by planar whole-body scintigraphy. This could lead to an underestimation of tWBS diagnostic value in this study $(18,19$, 20). An abnormal POCUS is highly predictive of PD with high sensitivity, especially when combined with RAI-Tg levels. Rosario et al.'s results $(12,21)$ showed that a RAI-Tg threshold value $>10 \mu \mathrm{g} / \mathrm{l}$ is predictive of $\mathrm{PD}$, but this report only included LR patients without initial LN metastasis. This finding should be taken with caution for patients with interfering anti-Tg antibodies. In our study, although some patients presented such antibodies, they were not excluded and RAI-Tg levels remained closely associated with the risk of recurrence.

Finally, in multivariate analysis, only the POCUS and the RAI-Tg results remain predictive of PD (Table 2). Neither the tWBS, nor any other classical risk factors such as initial pTNM, male sex, and age are significantly associated with PD. Likewise, initial metastatic status (19 patients) does not influence PD risk in multivariate analysis. We chose not to exclude these patients, considering that POCUS is a part of their management and its diagnostic value for PD and recurrence should be evaluated.

\section{POCUS contribution to patient management}

A normal POCUS enabled us to reassure 461 patients (70.7\%). Moreover, more than $95 \%$ of patients with a normal POCUS never developed a recurrence. This high NPV of POCUS when combined with an undetectable RAITg was also reported by Nascimento et al. (22) (whose series included $44.2 \%$ of $\mathrm{N} 1$ patients). The author used this high NPV in the RAI decision-making and follow-up.

Regarding TP POCUS results, patients were reoperated, on average, 13 months after POCUS. This time lapse can be explained by the necessity for a systematic US and FNAB confirmation 3-6 months after RAI treatment and for some patients because of the small size of suspect LN detected. Some series outline that $\mathrm{LN}$ reoperation achieves biochemical complete remission in only $27-51 \%$ of cases and sometimes requires several operations $(23,24)$ and other series support the fact that detecting $\mathrm{LN}$ after thyroidectomy for PTC is of no clinical importance (25). Although the long-term effectiveness of LN reoperation has not been proved in our study, it could alter the survival rate in patients with recurrence, especially for radioiodine refractory recurrence, located in the thyroid bed or in the neck soft tissues $(26,27)$. Whilst reoperation could be criticized, POCUS is necessary to identify patients with HR of recurrence and recent European US guidelines should help identify those requiring FNAB and surgery (14).
A false-positive POCUS result (64/638 (10\%) in our series) risks creating anxiety for both patients and clinicians and can lead to unnecessary examinations. Several factors could account for this rate: the(se) small LN metastases could have been destroyed by the RAI treatment or could remain dormant (25). Another explanation could be the timing of POCUS, which was carried out, on average, 90 days after surgery ( \pm 48 days). Too early, a POCUS could lead to the misinterpretation of post-surgery tissue swelling. In our study, we showed a better specificity of POCUS when the examination was done 3 months after surgery without modification of the sensitivity. However, among the 65 patients who had a suspect POCUS considered to be FP $(64 / 638 ; 10 \%)$, only 11 (1.7\% of the entire cohort) underwent a FNAB and two $(0.3 \%)$ had negative neck surgery.

Finally, based on the latest ATA guidelines (6) that recommend a preoperative neck US to assess the central and lateral neck compartments for all patients undergoing thyroidectomy for malignant cytology, one could argue the relevance of doing a POCUS if this preoperative US is done proficiently. In our series, a preoperative US was systematically done for all patients before surgery. However, because of the retrospective design of the study,

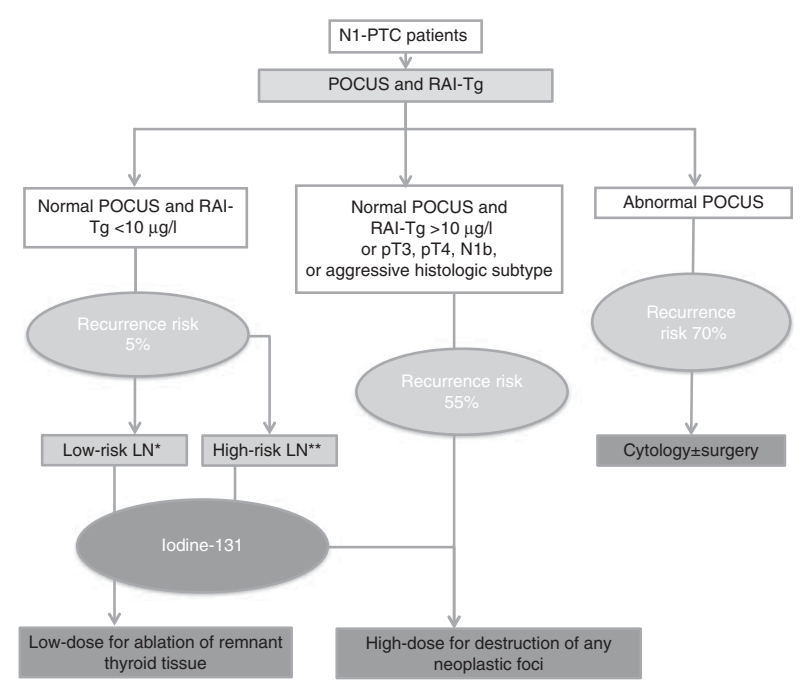

\section{Figure 2}

Proposed clinical algorithm according to POCUS and RAI-Tg results. N1-PTC, papillary thyroid cancer with initial lymph node involvement considered as high-risk recurrence according to ETA guidelines; POCUS, postoperative ultrasound; LN, lymph node; LN-ECI, extra-capsular LN involvement; low-risk LN*: less than five LN involved and no LNECl; high-risk-LN**: greater than or equal to five LN involved and/or LN-ECI. 
conducted from 1997 to 2010, a systematic second neck US following FNAB malignant results was not always performed as suggested in the recent recommendation and not always in a reference center. Furthermore, it has been reported that preoperative US identifies only half the number of LNs found at surgery, due to the presence of the overlying thyroid gland (28). Moreover, this POCUS can reveal LN metastases that were not detectable at surgery but whose growth had developed since due to the TSH stimulation required for radioiodine treatment.

\section{POCUS contribution to risk reassessment}

$\mathrm{N} 1$ patients have recently been shown as a heterogeneous group regarding recurrence risk (29), since the location of LN involvement (central N1a vs lateral N1b), the existence of LN-ECI, the size and number of initial LN metastasis $(29,30)$ are all factors that determine this risk. Recently, some authors have introduced a new concept of delayed risk stratification (DRS) based on data obtained 8-12 months after RAI, the time of the first diagnostic control $(5,9)$. This DRS better defined the patient's risk of recurrence and interestingly (DRS) led to a significant change in risk estimates in N1-PTC patients (5).

In our series, N1a patients without N1-ECI showed a lower risk of PD than that of other LN (Table 2). Therefore, early risk stratification could be created by re-stratifying N1-PTC patients with normal POCUS from intermediate to LR of PD according to the ATA classification. Indeed, only $3.2 \%(21 / 482)$ of patients with a negative POCUS developed a recurrence during the first year of follow-up. Furthermore, a combination of normal POCUS and RAI-Tg value under $10 \mu \mathrm{g} / \mathrm{l}$ conferred a 5-year cumulative incidence risk of recurrence of $<6 \%$ whatever the initial pTNM group. Thus, the recurrence risk is comparable with the ATA LR group PTC $(5,6)$.

Consequently, early risk reassessment with POCUS could help to individually tailor radioiodine treatment to individual needs. As suggested in Fig. 2, patients with a normal POCUS and RAI-Tg $<10 \mu \mathrm{g} / \mathrm{l}$ present less risk of recurrence $(5 \%)$ and could be treated with a low dose (1.1 GBq) of radioiodine as has been suggested in three recent studies $(31,32,33)$. However, other patients could benefit from a more traditional radioiodine treatment in order to destroy any neoplastic foci.

In conclusion, despite some limitations inherent to its retrospective design (about 10\% patients lost during followup, impossibility to isolate the subgroup of ATA intermediate pT3 subgroup with microscopic perithyroidal invasion), the results of this large cohort study highlight the crucial diagnostic role of POCUS in detection of PD. A normal POCUS result combined with a low RAI-Tg level allows the clinician to reassure the patient by re-stratifying his case into the LR N1-PTC category. Thus, our results support the introduction of a systematic POCUS in individualized risk assessment to improve therapeutic interventions and follow-up management.

\section{Declaration of interest}

The authors declare that there is no conflict of interest that could be perceived as prejudicing the impartiality of the research reported.

\section{Funding}

This research did not receive any specific grant from any funding agency in the public, commercial or not-for-profit sector.

\section{References}

1 Leboulleux S, Rubino C, Baudin E, Caillou B, Hartl DM, Bidart JM, Travagli JP \& Schlumberger M. Prognostic factors for persistent or recurrent disease of papillary thyroid carcinoma with neck lymph node metastases and/or tumor extension beyond the thyroid capsule at initial diagnosis. Journal of Clinical Endocrinology and Metabolism 2005 90 5723-5729. (doi:10.1210/jc.2005-0285)

2 Mazzaferri EL \& Jhiang SM. Long-term impact of initial surgical and medical therapy on papillary and follicular thyroid cancer. American Journal of Medicine 199497 418-428. (doi:10.1016/00029343(94)90321-2)

3 Schlumberger MJ. Papillary and follicular thyroid carcinoma. New England Journal of Medicine 1998338 297-306. (doi:10.1056/ NEJM199801293380506)

4 Mazzaferri EL. An overview of the management of papillary and follicular thyroid carcinoma. Thyroid 19999 421-427. (doi:10.1089/ thy.1999.9.421)

5 Tuttle RM, Tala H, Shah J, Leboeuf R, Ghossein R, Gonen M, Brokhin M, Omry G, Fagin JA \& Shaha A. Estimating risk of recurrence in differentiated thyroid cancer after total thyroidectomy and radioactive iodine remnant ablation: using response to therapy variables to modify the initial risk estimates predicted by the new American Thyroid Association staging system. Thyroid 201220 1341-1349. (doi:10.1089/ thy.2010.0178)

6 Cooper DS, Doherty GM, Haugen BR, Kloos RT, Lee SL, Mandel SJ, Mazzaferri EL, McIver B, Pacini F, Schlumberger M et al. Revised American Thyroid Association management guidelines for patients with thyroid nodules and differentiated thyroid cancer. Thyroid 2009 19 1167-1214. (doi:10.1089/thy.2009.0110)

7 Pacini F, Schlumberger M, Dralle H, Elisei R, Smit JW \& Wiersinga W. European consensus for the management of patients with differentiated thyroid carcinoma of the follicular epithelium. European Journal of Endocrinology 2006154 787-803. (doi:10.1530/eje.1.02158)

8 Borson-Chazot F, Bardet S, Bournaud C, Conte-Devolx B, Corone C, D'Herbomez M, Henry JF, Leenhardt L, Peix JL, Schlumberger M et al. Guidelines for the management of differentiated thyroid carcinomas of vesicular origin. Annales d'Endocrinologie 200869 472-486. (doi:10.1016/j.ando.2008.10.002)

9 Castagna MG, Maino F, Cipri C, Belardini V, Theodoropoulou A, Cevenini G \& Pacini F. Delayed risk stratification, to include the response to initial treatment (surgery and radioiodine ablation), has better outcome predictivity in differentiated thyroid cancer patients. 
European Journal of Endocrinology 2011165 441-446. (doi:10.1530/ EJE-11-0466)

10 de Rosario PW, Guimaraes VC, Maia FF, Fagundes TA, Purisch S, Padrao EL, Rezende LL \& Barroso AL. Thyroglobulin before ablation and correlation with posttreatment scanning. Laryngoscope 2005115 264-267. (doi:10.1097/01.mlg.0000154730.31281.0c)

11 Vaisman A, Orlov S, Yip J, Hu C, Lim T, Dowar M, Freeman JL \& Walfish PG. Application of post-surgical stimulated thyroglobulin for radioiodine remnant ablation selection in low-risk papillary thyroid carcinoma. Head \& Neck 201032 689-698. (doi:10.1002/hed.21371)

12 Rosario PW, Xavier AC \& Calsolari MR. Value of postoperative thyroglobulin and ultrasonography for the indication of ablation and ${ }^{131}$ I activity in patients with thyroid cancer and low risk of recurrence. Thyroid 201121 49-53. (doi:10.1089/thy.2010.0145)

13 Haber RS. Role of ultrasonography in the diagnosis and management of thyroid cancer. Endocrine Practice 20006 396-400. (doi:10.4158/ EP.6.5.396)

14 Leenhardt L, Erdogan MF, Hegedus L, Mandel SJ, Paschke R, Rago T \& Russ G. European Thyroid Association guidelines for cervical ultrasound-guided techniques in the postoperative management of patients with thyroid cancer. European Thyroid Journal 20132 147-159. (doi:10.1159/000354537)

15 Kim MJ, Kim EK, Kim BM, Kwak JY, Lee EJ, Park CS, Cheong WY \& Nam KH. Thyroglobulin measurement in fine-needle aspirate washouts: the criteria for neck node dissection for patients with thyroid cancer. Clinical Endocrinology 200970 145-151. (doi:10.1111/j. 1365-2265.2008.03297.x)

16 Pacini F, Capezzone M, Elisei R, Ceccarelli C, Taddei D \& Pinchera A. Diagnostic 131-iodine whole-body scan may be avoided in thyroid cancer patients who have undetectable stimulated serum Tg levels after initial treatment. Journal of Clinical Endocrinology and Metabolism 2002 87 1499-1501. (doi:10.1210/jcem.87.4.8274)

17 Cailleux AF, Baudin E, Travagli JP, Ricard M \& Schlumberger M. Is diagnostic iodine-131 scanning useful after total thyroid ablation for differentiated thyroid cancer? Journal of Clinical Endocrinology and Metabolism 200085 175-178. (doi:10.1210/jcem.85.1.6310)

18 Chen L, Luo Q, Shen Y, Yu Y, Yuan Z, Lu H \& Zhu R. Incremental value of ${ }^{131}$ I SPECT/CT in the management of patients with differentiated thyroid carcinoma. Journal of Nuclear Medicine 200849 1952-1957. (doi:10.2967/jnumed.108.052399)

19 Spanu A, Solinas ME, Chessa F, Sanna D, Nuvoli S \& Madeddu G. ${ }^{131}$ I SPECT/CT, in the follow-up of differentiated thyroid carcinoma: incremental value versus planar imaging. Journal of Nuclear Medicine 200950 184-190. (doi:10.2967/jnumed.108.056572)

20 Ciappuccini R, Heutte N, Trzepla G, Rame JP, Vaur D, Aide N\& Bardet S. Postablation (131)I scintigraphy with neck and thorax SPECT-CT and stimulated serum thyroglobulin level predict the outcome of patients with differentiated thyroid cancer. European Journal of Endocrinology 2011164 961-969. (doi:10.1530/EJE-11-0156)

21 Gomez Hernandez K, Etarsky D, Orlov S \& Walfish PG. Stimulated thyroglobulin and neck ultrasonography facilitates postsurgical radioactive iodine remnant ablation selection in patients with low-risk well-differentiated thyroid carcinoma. Thyroid 201222 760-761. (doi:10.1089/thy.2011.0453)

22 Nascimento C, Borget I, Al Ghuzlan A, Deandreis D, Chami L, Travagli JP, Hartl D, Lumbroso J, Chougnet C, Lacroix L et al. Persistent disease and recurrence in differentiated thyroid cancer patients with undetectable postoperative stimulated thyroglobulin level. Endocrine-Related Cancer 201118 R29-R40. (doi:10.1677/ERC-10-0292)

23 Al-Saif O, Farrar WB, Bloomston M, Porter K, Ringel MD \& Kloos RT. Long-term efficacy of lymph node reoperation for persistent papillary thyroid cancer. Journal of Clinical Endocrinology and Metabolism 201095 2187-2194. (doi:10.1210/jc.2010-0063)

24 Yim JH, Kim WB, Kim EY, Kim WG, Kim TY, Ryu JS, Gong G, Hong SJ \& Shong YK. The outcomes of first reoperation for locoregionally recurrent/persistent papillary thyroid carcinoma in patients who initially underwent total thyroidectomy and remnant ablation. Journal of Clinical Endocrinology and Metabolism 201196 2049-2056. (doi:10.1210/jc.2010-2298)

25 Robenshtok E, Fish S, Bach A, Dominguez JM, Shaha A \& Tuttle RM. Suspicious cervical lymph nodes detected after thyroidectomy for papillary thyroid cancer usually remain stable over years in properly selected patients. Journal of Clinical Endocrinology and Metabolism 2012 97 2706-2713. (doi:10.1210/jc.2012-1553)

26 Rouxel A, Hejblum G, Bernier MO, Boelle PY, Menegaux F, Mansour G, Hoang C, Aurengo A \& Leenhardt L. Prognostic factors associated with the survival of patients developing loco-regional recurrences of differentiated thyroid carcinomas. Journal of Clinical Endocrinology and Metabolism 200489 5362-5368. (doi:10.1210/jc.2003-032004)

27 Mazzaferri EL \& Kloos RT. Clinical review 128: Current approaches to primary therapy for papillary and follicular thyroid cancer. Journal of Clinical Endocrinology and Metabolism 200186 1447-1463. (doi:10.1210/ jcem.86.4.7407)

28 Leboulleux S, Girard E, Rose M, Travagli JP, Sabbah N, Caillou B, Hartl DM, Lassau N, Baudin E \& Schlumberger M. Ultrasound criteria of malignancy for cervical lymph nodes in patients followed up for differentiated thyroid cancer. Journal of Clinical Endocrinology and Metabolism 200792 3590-3594. (doi:10.1210/jc.2007-0444)

29 Randolph G, Duh QY, Heller KS, Livolsi VA, Mandel SJ, Steward D, Tufano RP \& Tuttle RM. The prognostic significance of nodal metastases from papillary thyroid carcinoma can be stratified based on the size and number of metastatic lymph nodes, as well as the presence of extranodal extension ATA surgical affairs committee's taskforce on thyroid cancer nodal surgery. Thyroid 201222 1144-1152. (doi:10.1089/thy.2012.0043)

30 Bardet S, Malville E, Rame JP, Babin E, Samama G, De Raucourt D, Michels JJ, Reznik Y \& Henry-Amar M. Macroscopic lymph-node involvement and neck dissection predict lymph-node recurrence in papillary thyroid carcinoma. European Journal of Endocrinology 2008158 551-560. (doi:10.1530/EJE-07-0603)

31 Mallick U, Harmer C, Yap B, Wadsley J, Clarke S, Moss L, Nicol A, Clark PM, Farnell K, McCready R et al. Ablation with low-dose radioiodine and thyrotropin alfa in thyroid cancer. New England Journal of Medicine 2012366 1674-1685. (doi:10.1056/ NEJMoa1109589)

32 Schlumberger M, Catargi B, Borget I, Deandreis D, Zerdoud S, Bridji B, Bardet S, Leenhardt L, Bastie D, Schvartz C et al. Strategies of radioiodine ablation in patients with low-risk thyroid cancer. New England Journal of Medicine 2012366 1663-1673. (doi:10.1056/ NEJMoa1108586)

33 Rosario PW \& Calsolari MR. Thyroid ablation with $1.1 \mathrm{GBq}(30 \mathrm{mCi})$ iodine-131 in patients with papillary thyroid carcinoma at intermediate risk for recurrence. Thyroid 2014. In Press.
Received 31 October 2013

Revised version received 11 February 2014 Accepted 21 March 2014 